\title{
Musculoskeletal ultrasound guiding shoulder injection when "one picture is worth ten thousand landmarks"
}

\author{
Mihaela C. Micu', Annamaria Iagnocco² \\ ${ }^{1}$ Rheumatology Division, Department of Rehabilitation II, Rehabilitation Clinical Hospital Cluj-Napoca, Romania \\ ${ }^{2}$ Rheumatology Unit, Dipartimento Medicina Interna e Specialità Mediche, Sapienza Università di Roma, Rome, Italy
}

Over the last years, ultrasound guided procedures have shown a low incidence of adverse events $(<5 \%)$ with an excellent efficacy and safety profile [1]. This evidence suggests that ultrasound guidance achieves successful intrarticular injection of the medication avoiding adverse events and drug misplacement during the injection. Indeed, it is well known that the problem of acute musculoskeletal pain and its real time management are today sensitive issues in rheumatology practice.

Shoulder emergencies are characterized by acute pain and disability and comprise mainly of a large spectrum of periarticular pathology. Among these, the subacromiosubdeltoidian bursa is frequently involved, highly symptomatic and may represent, alone or in association with other periarticular modifications, the cause of an acute or chronic coraco-acromial impingement syndrome [2].

This kind of pathology is very frequently encountered in clinical practice and requires immediate medical help. A quick individualized, safe and feasible strategy, in terms of treatment efficacy as well as cost effectiveness, is desirable.

In the last years, the use of musculoskeletal ultrasound (MSUS) for shoulder evaluation has constantly shown clear superiority to clinical examination for diagnostic purposes. [3] In fact, MSUS is a highly sensitive and reliable technique that allows the identification of uni- or multistructural lesions at periarticular and/ or joint level [2-4]. Comparing MSUS with a high quality imaging method such as magnetic resonance imaging

Received Accepted

Med Ultrason

2012, Vol. 14, No 4, 3-5

Corresponding author: Mihaela C. Micu,

Division of Rheumatology,

Rehabilitation Department II

Rehabilitation Clinical Hospital

46-50 Viilor str, Cluj- Napoca, Romania

Email:mcmicu@yahoo.com
(MRI), showed similar sensitivity in detecting periarticular shoulder pathology and an additional value was found for MRI only for the detection of intraarticular lesions [5]. A very well known advantage of MSUS over MRI is the possibility to perform, at bedside, a real time dynamic evaluation. Moreover, according to site and type of periarticular lesions, MSUS may offer information that can predict the outcome in inflammatory as well as degenerative pathology [6]. These characteristics identify MSUS as a tool which allows the future design of short and long term treatment strategies. Being at the same time a low cost imaging method, non invasive and non x-ray burdened, it has been implemented in daily clinical practice in rheumatology units as a diagnostic tool and for guiding interventional maneuvers $[2,7,8]$.

In subacromio-subdeltoidian bursitis, MSUS allows the evaluation of the bursal wall and content- effusion and / or synovial hypertrophy, dimensions and extension as well as the bursal movement and coraco- acromial encroachment while performing dynamic examination of the rotator cuff. MSUS is the ideal imaging method for precise bursal real time punction under continuous needle guidance followed by accurate drug deposition $[2,8]$.

In the last years, attention has been focused mainly on local treatment efficacy, representing the final goal of the medical visit. If local MSUS guided treatment offers supplementary advantages over blind drug deposition, it remains a subject still intensely discussed and under debate. Despite important available medical data concerning the substantial advantages in achieving accuracy/ efficacy of imaging guided drug deposition $[7,8]$, there are still supporters for performing blind shoulder injections. The main reasons for this attitude are the need of supplementary costs / time for performing imaging guided procedures and data showing similar therapeutic outcome when comparing results of local imaging guided injec- 


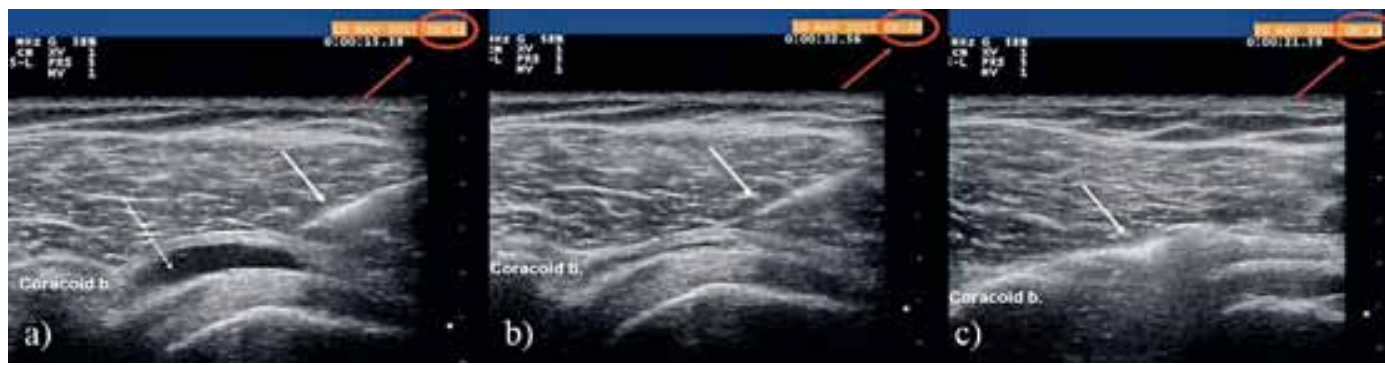

Fig 1. Anterior transversal scan of the shoulder. a) effusion in the subacromio-subdeltoidian bursa (punctured white arrow); white arrow-; the needle; b) after bursal content aspiration; white arrow-; the needle; c) corticosteroids deposited inside the bursa - the hyperechoic mass generating acoustic shadow artefact (white arrow); coracoid b- coracoid bone. Time display -red circle and red arrow.

tions and systemic administration or local blind versus guided drug deposition $[9,10]$.

We agree that therapeutic results can be obtained, sometimes satisfactorily, by blindly placing corticosteroids (CS) in the shoulder area. Beside the correct blind intrabursal deposition using bony landmarks, also the peribursal deposition may be of benefit because of the CS diffusion capacity in the surrounding tissues, including the bursa. If the drug deposition misses the bursa, it can be placed inside the deltoid muscle or inside the rotator cuff tendons. In the first case, CS deposition functions like an ordinary intramuscular CS injection being equivalent to systemic administration but subsequently exposing to known side effects, whereas in the second case, minor tendon injuries up to more serious such as tendon rupture may occur [11].

A sometimes neglected but very important issue is the subdeltoidian bursal aspiration, not only for diagnostic purposes (macroscopy- synovial fluid / blood; microscopy- cytology, crystals, gram stain/ tuberculosis) but also, because of mechanical decompression with immediate reduction of the impingement syndrome. On the other hand, chronic persistency of intrabursal effusion/ synovial hypertrophy exposes to surrounding tissue destruction due to continuous traumatic injury by the subcoraco- acromial encroachment and due to the inflammatory products content [4].

In complex anatomical areas such as the shoulder, blind aspiration is rarely successful and current evidence shows that even in areas with easier access, blind aspiration has not always been a valuable predictor of correct target needle placement [12].

Efficient, complete aspiration needs precise needle placement under imaging guidance. This maneuver may be followed by correct intrabursal CS deposition with rapid pain reduction, avoiding chronic exposure to nonsteroid-anti-inflammatory drugs and potential digestive, cardio-vascular and renal side effects, prolonged physical therapy programs with additional costs and delayed results.

In fact, in just one minute, under MSUS guidance, free hand technique, subdeltoid bursa can be punctured, effusion evacuated and CS safely deposited (fig 1) The great advantage in using MSUS for guided diagnostic and therapeutic injections relies on the freedom to position the shoulder, arm and forearm in order to expose best the target area having real time control, to choose the shortest way from skin to the target and the most convenient position for the patient and performing doctor. Being a non-radiant method, no supplementary protection measures are needed.

After wearing sterile gloves and proper skin disinfection, nonsterile gel is placed strictly under the transducer footprint and the needle (18-22 gauge) is inserted in a "free hand" technique through the skin at $1 \mathrm{~cm}$ distance far away from the probe with an angulation of $45^{\circ}$ to the horizontal plane, inside the ultrasound beam. The needle is identified as a hyperechoic line, generating sometimes comet tail artifact. It penetrates the subcutaneous tissue, deltoid muscle, bursal wall and reaches the bursa. At this point, aspiration and drug deposition can be quickly and accurately made [8].

In conclusion, it is always worth being quick, safe and accurate avoiding blind procedures that may harm the patient and result in further complications afterwards. It is really time to take this issue seriously and teach our fellows to prefer the guided maneuvers avoiding as much as possible the blind ones. The help of MSUS, in real time at the patient's bedside, supports, in rheumatologic practice, the Chinese proverb that "one picture is worth ten thousand words". This means that our hands hold the success of our therapies and we rheumatologists should never miss the guiding opportunity which the new imaging MSUS frontier is offering us. 


\section{References}

1. Migliore A, Massafra U, Bizzi E, et al. Comparative, double-blind, controlled study of intra-articular hyaluronic acid (Hyalubrix) injections versus local anesthetic in osteoarthritis of the hip. Arthritis Res Ther 2009; 11: R183.

2. Iagnocco A, Filippucci E, Meenagh G, et al. Ultrasound imaging for the rheumatologist I. Ultrasonography of the shoulder. Clin Exp Rheumatol 2006; 24: 6-11.

3. Naredo E, Aguado P, De Miguel E, et al. Painful shoulder: comparison of physical examination and ultrasonographic findings. Ann Rheum Dis 2002; 61: 132-136.

4. Bruyn GA, Pineda C, Hernandez-Diaz C, et al. Validity of ultrasonography and measures of adult shoulder function and reliability of ultrasonography in detecting shoulder synovitis in patients with rheumatoid arthritis using magnetic resonance imaging as a gold standard. Arthritis Care Res (Hoboken) 2010; 62: 1079-1086.

5. Rutten MJ, Spaargaren GJ, Van Loon T, De Waal Malefijt MC, Kiemeney LA, Jager GJ. Detection of rotator cuff tears: the value of MRI following ultrasound. Eur Radiol 2010; 20: 450-457.

6. Naredo E, Collado P, Cruz A, et al. Longitudinal power Doppler ultrasonographic assessment of joint inflamma- tory activity in early rheumatoid arthritis: predictive value in disease activity and radiologic progression. Arthritis Rheum 2007; 57: 116-124.

7. Naredo E, Cabero F, Cruz A, Uson J, Palop MJ, Crespo M. Ultrasound guided musculoskeletal injections. Ann Rheum Dis 2005; 64: 341.

8. Naredo E, Cabrero F, Beneyto $\mathrm{P}$, et al. A randomized comparative study of short term response to blind injection versus sonographyc- guided injection of local corticosteroids in patients with painful shoulder. J Rheumatol 2004; 31 : 308-314.

9. Hall S, Buchbinder R. Do imaging methods that guide needle placement improve outcome? Ann Rheum Dis 2004; 63: 1007-1008.

10. Rutten MJ, Maresch BJ, Jager GJ, De Waal_Malefijt MC. Injection of the subacromial -subdeltoid bursa: blind or ultrasound-guided? Acta Orthop 2007; 78: 254-257.

11. Canoso JJ. Aspiration and injection of joints and periarticular tissues (including articular and intralesional therapy). In: Hochberg MC, Silman AJ, Smolen JS, Weinblatt ME, Weisman MMH. Rheumatology, Mosby Elsevier 2008: 421-432.

12. Jones A, Reagan M, Ledingham J, Patrick M, Manhire A, Doherty M. Importance of placement of intra-articular steroid injections. BMJ 1993; 20: 1329-1330. 